Article

\title{
Improving Absorption in Single Silicon Nanowires by Symmetry-breaking Design from Square to Rectangular Cross-section
}

\author{
Wenfu Liu ${ }^{1, *}$ \\ ${ }^{1}$ School of Energy Engineering, Huanghuai University, Zhumadian, Henan 463000, China \\ * Correspondence: liuwenfu@huanghuai.edu.cn
}

\begin{abstract}
Light absorption in single nanowires (NWs) is one of the most crucial factors for photovoltaic applications. In this paper, we carried out a detailed investigation of light absorption in single rectangular NWs (RNWs). We show that the RNWs exhibit improved light absorption compared with the square NWs (SNWs), which can be attributed to the symmetry-breaking structure that can increase the light path length by increasing the vertical side and the enhanced leaky mode resonances (LMRs) by decreasing the horizontal side. We found that the light absorption in silicon RNWs can be enhanced by engineering the horizontal and vertical sides, the photocurrent is significantly increased by $276.5 \%$ or $82.9 \%$ compared with that of the SNWs with the same side length as the horizontal side of $100 \mathrm{~nm}$ or the vertical side of $1000 \mathrm{~nm}$, respectively. This work provides an effective way for designing high-efficiency single NW photovoltaic devices based on the symmetry breaking from the SNWs to RNWs.
\end{abstract}

Keywords: silicon; single nanowires; Symmetry breaking; rectangular cross-section; absorption

\section{Introduction}

Single nanowire (NW) solar cells have attracted more and more attention in recent years serving as powering nanoscale devices [1-8]. Improving light absorption is an effective way to enhance the photoelectric conversion efficiency of single NW solar cell. It is well known that light absorption in single NWs is significantly enhanced due to the leaky mode resonances (LMRs) [9-11]. However, the light absorption in single NWs is still far below expectation.

It has been shown that the light absorption could be increased by engineering the size, geometry, and orientation of the NWs [12-18]. We have also shown that the light absorption could be further enhanced by introducing a single shell $[19,20]$ or graded dual shells [21]. Recently, some new strategies have been performed to improve the light absorption in the NWs based on the symmetry breaking, such as opening crescent design [22,23], off-axial core-shell design [24,25], asymmetrical nanovoid design [14], partially capped design [26-29] and crescent nanohole design [30]. More recently, we investigated the symmetry-breaking structure from circular NWs to elliptical NWs [31]. However, the light absorption in single NWs based on the symmetry-broken structure from the square NWs (SNWs) to rectangular NWs (RNWs) has rarely been explored so far.

In this paper, we present a detailed study on the light absorption in single RNWs. We show that the light absorption is enhanced when the cross-section of NWs is changed from square to rectangle. The results show the light path length can be increased by increasing the vertical side and the LMR modes can be enhanced by decreasing the horizontal side. The results reveal that the photocurrent is significantly enhanced by $276.5 \%$ or $82.9 \%$ compared with that of the SNWs with the same side length as the horizontal side of $100 \mathrm{~nm}$ or the vertical side of $1000 \mathrm{~nm}$, respectively. 


\section{Model and Methods}

In Figure 1, we show schematics of the cross-section of a RNW and two SNWs. The horizontal $(x)$ and vertical $(y)$ axes of the RNWs are labeled by $a$ and $b$. The light illumination direction is perpendicular (or parallel) to the horizontal (or vertical) side, as presented using the colorful arrows. Note here that the unpolarized light (e.g., sunlight) can be expressed as the average of transverse electric (TE, electric field normal to the NW axis) and transverse magnetic (TM, magnetic field normal to the NW axis) light, and the wavelength range of the incident light is from 300 to $1100 \mathrm{~nm}$ with a step size of $5 \mathrm{~nm}$ considering solar radiation and the bandgap of silicon. The values of $a$ and $b$ are chosen to be 100 and $300 \mathrm{~nm}$ as the typical representative nanoscale size, respectively. It is worth noting that the SNWs are also shown for comparison, where the side lengths of the SNWs are chosen to be 300 and 100 to investigate the improved light absorption due to the enhanced LMRs by decreasing $a$ and the increased light path length by increasing $b$. The material is chosen to be silicon and its complex refractive index is taken from the ref. [32]. The electric field inside the RNWs is calculated by two dimensional (2D) FDTD simulation by solving Maxwell's equations [33-35], Also, the absorption mode profile, the absorption efficiency, the photogeneration rate, and the ultimate photocurrent can be obtained $[21,31]$.

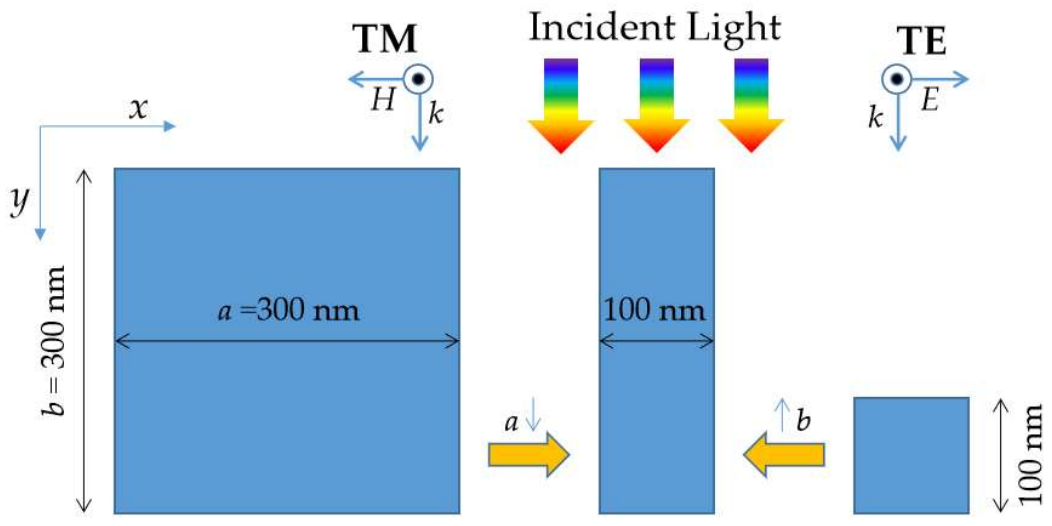

Figure 1. Cross-sectional views of a rectangular nanowire (RNW) and two square nanowires (SNWs). Representative values of the horizontal $(x)$ side $a$ and the vertical $(y)$ side $b$ of the RNW are chosen to be 100 and $300 \mathrm{~nm}$, respectively. The side lengths of the SNWs are chosen to be 300 or $100 \mathrm{~nm}$ for comparison, the material of the RNW is set to be silicon as a typical semiconductor and the light illumination is normal to the horizontal side of the RNW from above. Note that the unpolarized light (e.g., sunlight) illumination can be regarded as the average of transverse electric (TE, electric field normal to the NW axis) and transverse magnetic (TM, magnetic field normal to the NW axis) light illumination.

\section{Results and Discussion}

In Figure 2, we show $\lambda$-dependent $Q_{\text {abs }}$ spectra of the SNW1 with $a=b=300 \mathrm{~nm}$, the RNW with $a=100$ and $b=300 \mathrm{~nm}$ and the SNW2 with $a=b=100 \mathrm{~nm}$ under TM, TE and unpolarized light, respectively. Firstly, $Q_{\text {abs }}$ of the RNW is much bigger than that of the SNW2 almost the whole wavelength range, except for $560<\lambda<615 \mathrm{~nm}$ near the 3rd absorption peak of the SNW2 for TM light, $430<\lambda<455 \mathrm{~nm}$ near the $2 \mathrm{nd}$ absorption peak of the SNW2 for TE light and $560<\lambda<610 \mathrm{~nm}$ for near the 3rd absorption peak of the SNW2 for unpolarized light, which can lead to a significant photocurrent enhancement. Secondly, $Q_{\text {abs }}$ of the RNW is much bigger than that of the SNW1 in the short-wavelength range of $\lambda<\lambda_{\mathrm{cTM}} \sim 490, \lambda<\lambda_{\mathrm{cTE}} \sim 425$ or $\lambda<\lambda_{\mathrm{c}} \sim 485 \mathrm{~nm}$ for TM, TE or unpolarized light, which can result in a significant photocurrent enhancement. In contrast, the light absorption of the RNW seems to be comparable in the long-wavelength range of $\lambda>\lambda_{\text {cTM }} \lambda>\lambda_{\text {cTE }}$ or $\lambda>\lambda_{\mathrm{c}}$, which can lead to a little contribution to the photocurrent enhancement. Note here that $\lambda_{\mathrm{cTM}}, \lambda_{\mathrm{cTE}}$, and $\lambda_{\mathrm{c}}$ are the characteristic wavelengths for TM, TE, and unpolarized light, below which the light absorption is always increased and can be readily fixed by $a$ and $b$. More importantly, the $Q_{\text {abs }}$ spectra exhibit 
two strong absorption peaks in the wavelength range of $\lambda<\lambda_{\text {cTM }}$ or $\lambda<\lambda_{\text {cTE }}$ for TM or TE light, respectively. Specifically, the $Q_{\mathrm{abs}}$ value reaches 2.71 near $\lambda=440 \mathrm{~nm}$ for TM light and 2.14 near $\lambda=$ $395 \mathrm{~nm}$ for TE light, which results in a dramatic photocurrent enhancement. Note here that some $Q_{\mathrm{abs}}$ values exceed unity, which is ascribed to the fact that the absorption cross-section is bigger than the physical cross-section. Therefore, the RNWs have huge potential in improving light absorption owing to the NW's symmetry-breaking structure from the square to the rectangular cross-section.

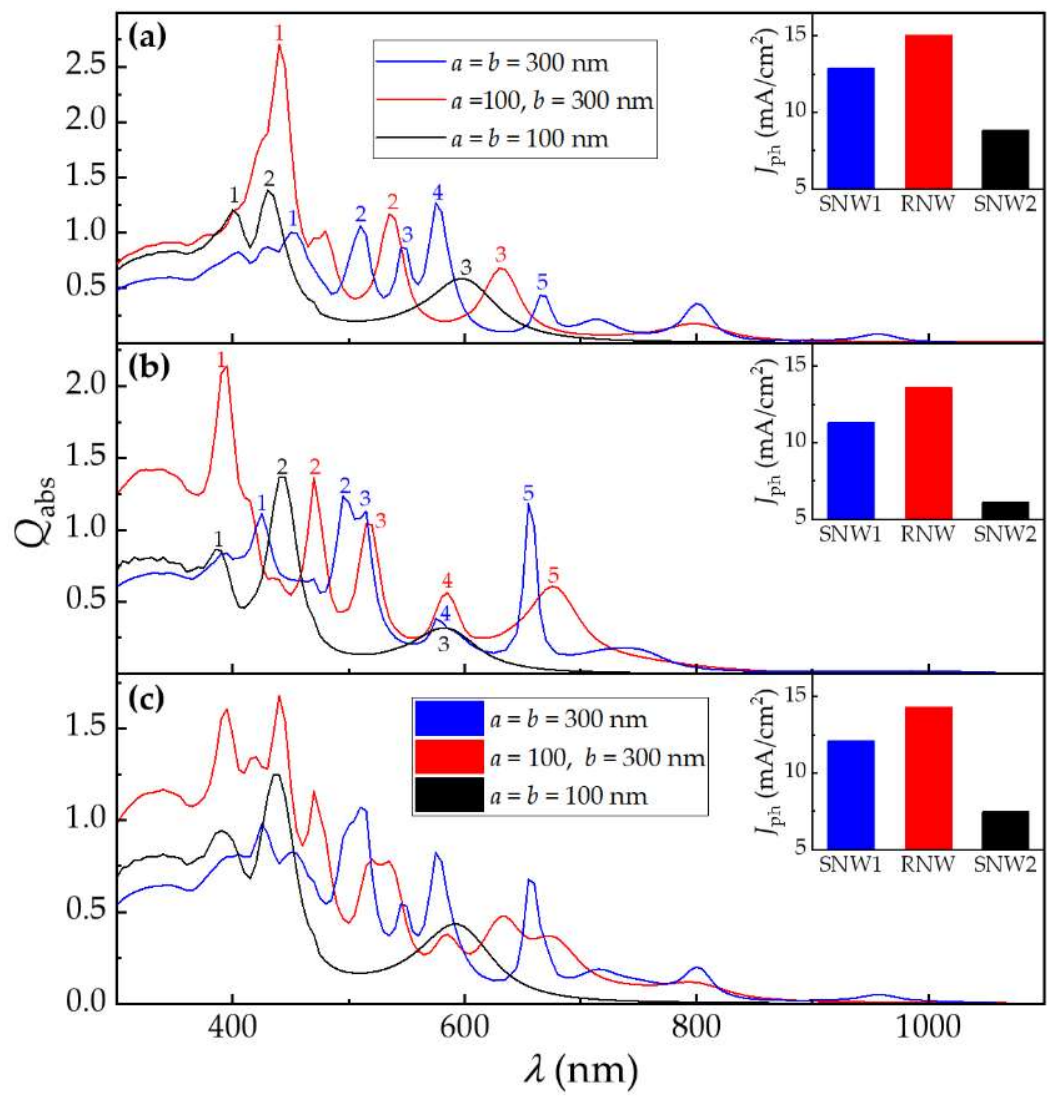

Figure 2. Absorption efficiency of the SNW1, RNW, and SNW2 as a function of wavelength for (a) $\mathrm{TM}$, (b) TE, and (c) unpolarized light illumination, respectively. The insets at the top right corner show the ultimate photocurrent of the SNW1, RNW, and SNW2 for (a) TM, (b) TE and (c) unpolarized light, respectively. Note that $a=100$ and $b=300 \mathrm{~nm}$.

In the insets of the top right corner of Figure 2, we show the ultimate photocurrent $J_{\mathrm{ph}}$ of the RNW and SNWs for TM, TE, and unpolarized light illumination, respectively. On one hand, the value of $J_{\mathrm{ph}}$ of the RNW is much larger than those of the SNW1 and SNW2. Jph reaches 15.07, 13.62 and 14.35 $\mathrm{mA} / \mathrm{cm}^{2}$, which is $17.00 \%, 20.42 \%$ and $18.69 \%$ higher than that of the SNW1 $(12.88,11.31$ and 12.09 $\left.\mathrm{mA} / \mathrm{cm}^{2}\right)$, respectively. Note here that the photocurrent enhancement is mainly attributed to the improved LMRs due to the decrease of $a$ (here from 300 to $100 \mathrm{~nm}$ ) in contrast with the SNW1. On the other hand, $J_{\mathrm{ph}}$ can be dramatically increased due to the improved light path length by the increase of $b$ (here 100 to $300 \mathrm{~nm}$ ) compared with the SNW2. Jph is $70.5 \%, 121.8 \%$, and $91.6 \%$ bigger than that of the SNW2 $\left(8.84,6.14\right.$, and $\left.7.49 \mathrm{~mA} / \mathrm{cm}^{2}\right)$ for TM, TE, and unpolarized light illumination, respectively.

In Figure 3, we present the normalized electric field $\left(E_{r}\right)$ profiles of the SNW1, RNW, and SNW2 for typical absorption peaks in Figure 2a,b under TM and TE light illumination, respectively. Note that Figure 3a,d present the $E_{r}$ profiles of the SNW1 for TM $(\lambda=450,510,545,575$ and $665 \mathrm{~nm})$ and TE $(\lambda=425,495,515,575$ and $655 \mathrm{~nm})$ light illumination, Figure 3b,e illustrate those of the RNW for $\operatorname{TM}(\lambda=440,535$ and $630 \mathrm{~nm})$ and TE $(\lambda=395,470,515,585$ and $675 \mathrm{~nm})$ light illumination, while Figure 3c,f exhibit those of the SNW2 for TM $(\lambda=400,430$ and $595 \mathrm{~nm})$ and TE $(\lambda=385,445$ and 580 
$\mathrm{nm})$ light illumination, respectively. On the one hand, there are similar features of the $E_{r}$ profiles between the RNW and SNWs. Firstly, the improved light absorption of the RNW is ascribed to the excitation of the LMRs, likewise in SNW [9,10]. For instance, the $E_{r}$ profiles of the RNW in Figure $3 b(3)$ and Figure $3 e(5)$ show more characteristics of the TM12 and $\mathrm{TE}_{31}$ modes of the RNW, respectively. Secondly, both NWs illustrate much larger $E_{r}$ intensities in the long- than in the shortwavelength range. For example, the $E_{r}$ intensities for the RNW are much higher in Figure 3e(3-5) than Figure 3e(1-2) for TE light, likewise the elliptical NWs. On the other hand, the $E_{r}$ intensities of the resonant peaks in the short wavelength range are much bigger inside the whole RNW owing to the excitation of more complex LMRs (for example, Figure $3 b(1)$ for TM light, indicating the stronger interaction between the incident light and the RNW, resulting in a stronger absorption in comparison with the SNWs.

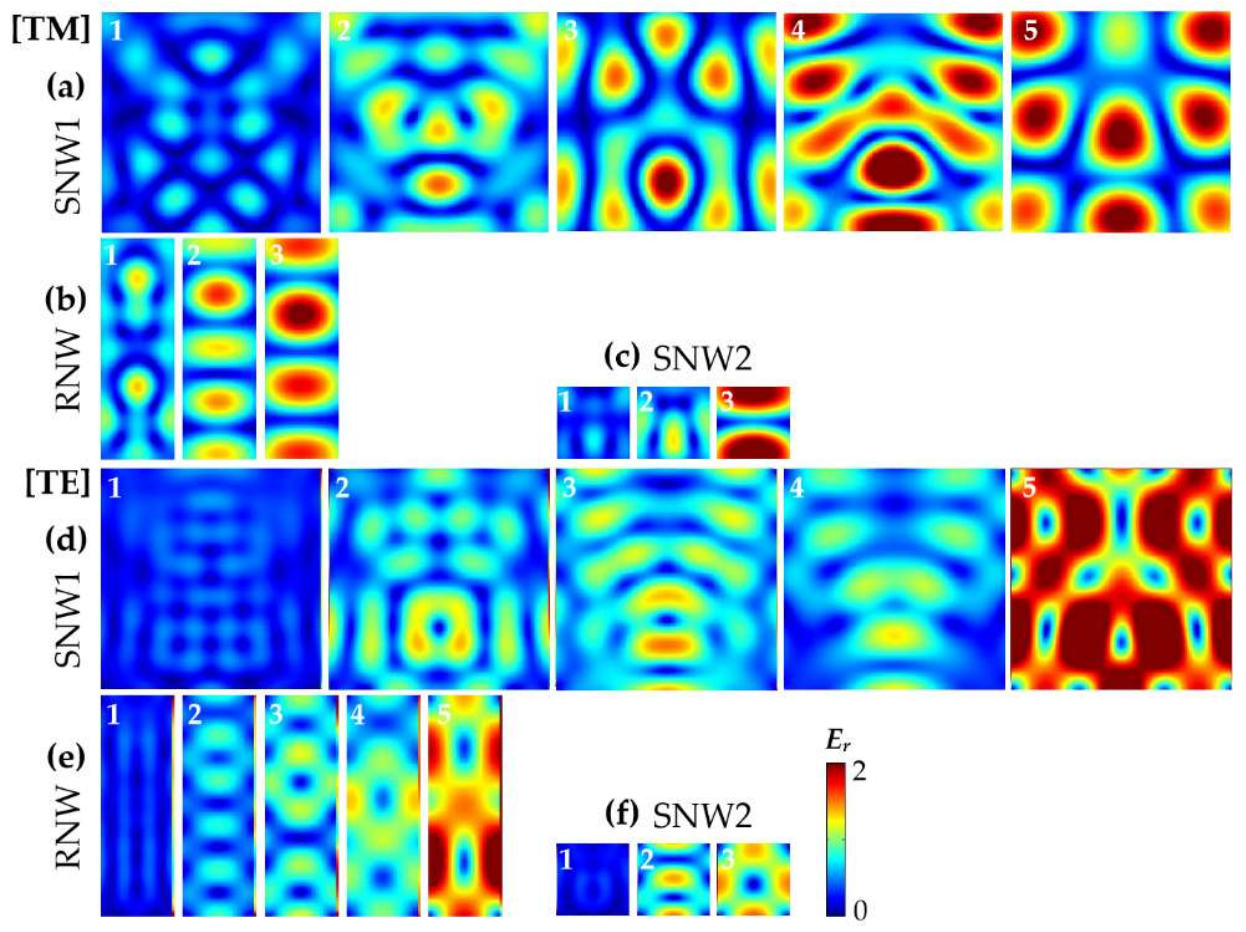

Figure 3. The typical normalized electric field profiles of the SNW1, RNW and SNW2 at the absorption peaks indicated by numerals in Figure $2 a, b:(\mathbf{a}, \mathbf{b}, \mathbf{c})$ for TM and $(\mathbf{d}, \mathbf{e}, \mathbf{f})$ for TE light illumination (with an identical color scale); (a,d) for the SNW1, $(\mathbf{b}, \mathbf{e})$ for the RNW and $(\mathbf{c}, \mathbf{f})$ for the SNW2, respectively.

In Figure 4, we show the corresponding normalized absorption mode profiles ( $P$ abs $)$ of the SNW1, RNW, and SNW2 under TM and TE light illumination, respectively. Note that Figure 4a,d present the normalized $P_{\text {abs }}$ of the SNW1 for TM and TE light, Figure 4b, exhibit those of the RNW for TM and TE light, while Figure 4c,f describe those of the SNW2, respectively. It is shown that the light absorption of the RNW in the short-wavelength range is much higher than that of the SNW2 (for example, Figure $3 b, e(1))$, leading to a significant photocurrent enhancement. Meanwhile, although the $P_{\text {abs }}$ intensities of the RNW in the short-wavelength range is comparable with that of the SNW2, the light path length of the RNW is three times as much as that of the SNWs, resulting in a dramatic photocurrent enhancement. 


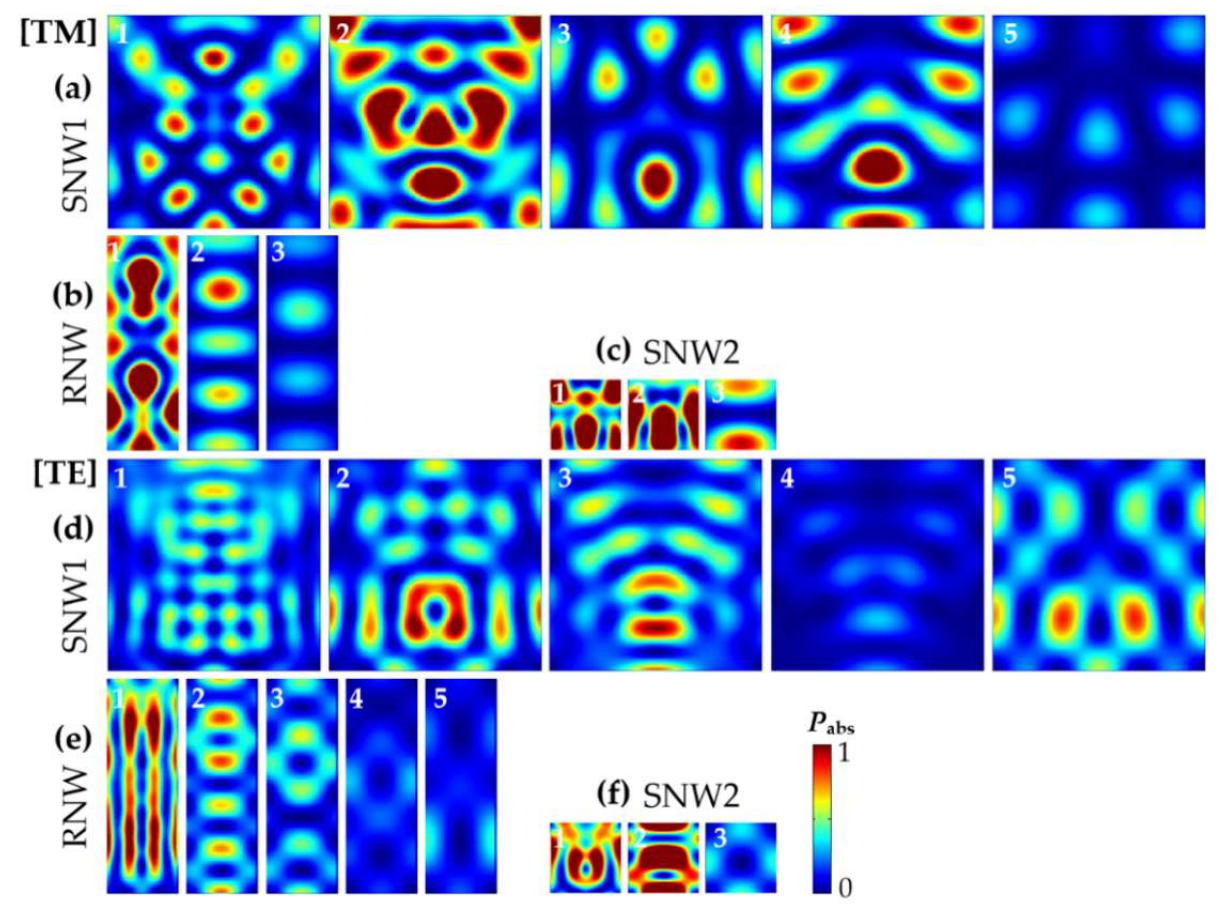

Figure 4. The representative normalized absorption mode profiles of the SNW1, RNW and SNW2 at the absorption peaks indicated by numerals in Figure $2 \mathrm{a}, \mathrm{b}:(\mathbf{a}, \mathbf{b}, \mathbf{c})$ for TM and $(\mathbf{d}, \mathbf{e}, \mathbf{f})$ for TE light illumination (with an identical color scale); (a,d) for the SNW1, (b,e) for the RNW and (c,f) for the SNW2, respectively.

In Figure 5, we show the normalized photogeneration rate $(G)$ profiles of the SNW1, RNW, and SNW2 for TM and TE light illumination, respectively. Note that Figure 5a,d illustrate the normalized $G$ profiles of the SNW1 for TM and TE light, Figure 5b,e present those of the RNW, while Figure 5c,f exhibit those of the SNW2, respectively. It is shown that the $G$ intensities of the RNW are much greater in the whole NW than those of the SNW1 for both TM and TE light. Note that although the G intensities of the RNW are slightly smaller than those of the SNW2 for both TM and TE light, the light path length is three times as much as that of the SNW2, which results in a giant photocurrent enhancement. These results further reveal that the light absorption is significantly enhanced due to the LMRs by decreasing $a$ compared to the SNW1 and enhanced light path length by increasing $b$.

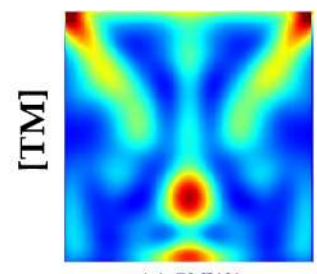

(a) SNW1

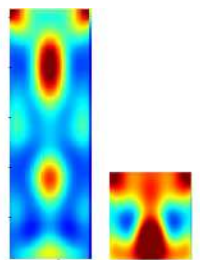

(b) RNW (c) SNW2

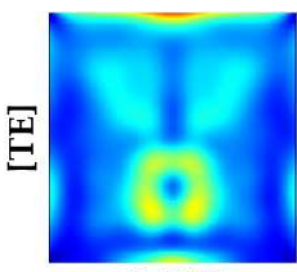

(d) SNW1

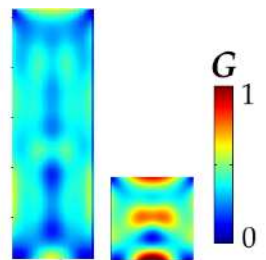

(e) RNW (f) SNW2

Figure 5. The normalized photogeneration rate profiles for SNW1, RNW and SNW2: $(a, b, c)$ for TM and $(\mathrm{d}, \mathrm{e}, \mathrm{f})$ for TE light illumination (with an identical color scale); $(\mathrm{a}, \mathrm{d})$ for the SNW1, $(\mathrm{b}, \mathrm{e})$ for the RNW and (c,f) for the SNW2, respectively.

In Figure 6a-c, we show 2D Jph maps as a function of $a$ (from 100 to $1000 \mathrm{~nm}$ ) and $b$ (from 100 to $1000 \mathrm{~nm}$ ) of the RNWs for TM, TE, and unpolarized light illumination, respectively. It is shown that we show $J_{\mathrm{ph}}$ sharply increases with decreasing $a$ at fixed $b$, reaches its maximum at $a=100 \mathrm{~nm}$, and $J_{\mathrm{ph}}$ also dramatically increases with increasing $b$ at a fixed $a$, reaches its maximum at $b=1000 \mathrm{~nm}$. More importantly, $J_{\mathrm{ph}}$ of the RNWs is always much larger than that of the SNWs at any $a(<b)$ values. 
It is observed that the maximum values of $J_{\mathrm{ph}}$ of the RNWs can be obtained in the length range of 100 $<a<300$ and $450<b<1000 \mathrm{~nm}$.

In Figure $6 \mathrm{~d}$,e, we show $J_{\mathrm{ph}}$ as a function of $a$ of the RNWs with $b=1000 \mathrm{~nm}$ and $J_{\mathrm{ph}}$ as a function of $b$ of the RNWs with $a=100 \mathrm{~nm}$ for TM, TE, and unpolarized light. We then in Figure 6f, g show the photocurrent enhancement factors (PEFs) defined by Equation (6). It is observed that $J_{\mathrm{ph}}$ of the RNWs increases with decreasing $a$ at a fixed $b=1000 \mathrm{~nm}$ and increasing $b$ at a fixed $a=100 \mathrm{~nm}$ for all polarized lights, reaches 26.90, 29.50 and $28.20 \mathrm{~mA} / \mathrm{cm}^{2}$ at $a=100$ and $b=1000 \mathrm{~nm}$, which is $81.0 \%$, $84.6 \%$ and $82.9 \%$ much larger than that of the SNW with $a=b=1000 \mathrm{~nm}(14.86,15.98$ and 15.42 $\left.\mathrm{mA} / \mathrm{cm}^{2}\right)$ and $204.3 \%, 380.5 \%$ and $276.5 \%$ much larger than that of the SNW with $a=b=100(8.84,6.14$ and $7.49 \mathrm{~mA} / \mathrm{cm}^{2}$ ) for TM, TE and unpolarized light, respectively.

Finally, we show in Figure 6h,i the normalized $G$ profiles of two SNWs and six RNWs for TM and TE light illumination, respectively. Note here that $a=1000,500,200$ and $100 \mathrm{~nm}$ at fixed $b=1000$ $\mathrm{nm}$ and $b=1000,500,200$ and $100 \mathrm{~nm}$ at a fixed $a=100 \mathrm{~nm}$, respectively. As shown in this figure, with the symmetry breaking from SNWs to RNWs $(a=1000 \rightarrow 500 \rightarrow 200 \rightarrow 100 \mathrm{~nm})$ with $b=1000 \mathrm{~nm}$, the LMR modes are reshaped due to the size decrease of the horizontal side, and the absorption enhancement sites better fill in the whole RNWs for both TM and TE lights, especially TE light. Meanwhile, with the decreased light path length from RNWs to SNWs $(b=1000 \rightarrow 500 \rightarrow 200 \rightarrow 100$ $\mathrm{nm}$ ) with $a=100 \mathrm{~nm}$, the photocurrent is decreased.
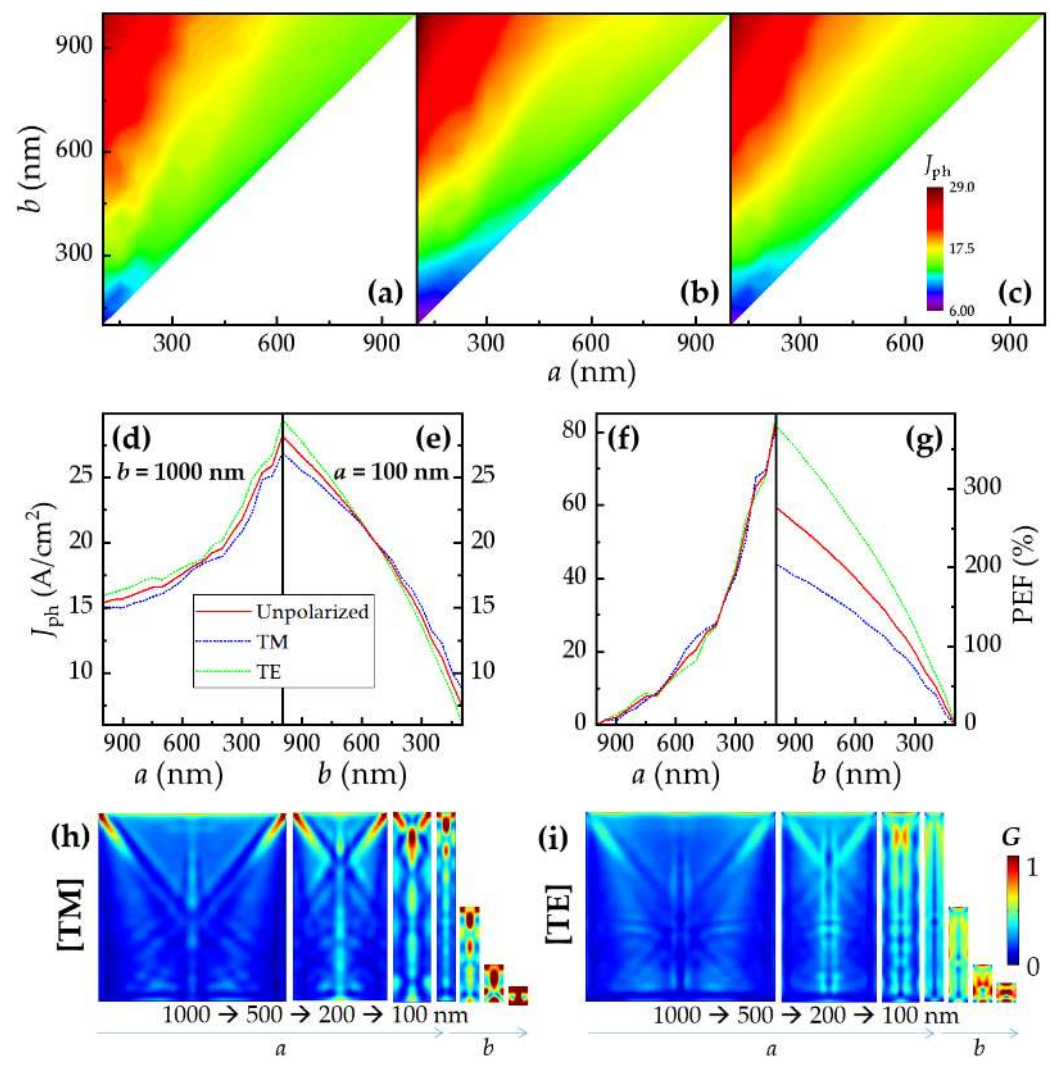

Figure 6. The ultimate photocurrent $\left(J_{\mathrm{ph}}\right)$ as a function of $a$ and $b$ of the RNWs for (a) TM, (b) TE and (c) unpolarized light illumination, respectively; (d) $J_{\mathrm{ph}}$ versus $a$ of the RNWs with $b=1000 \mathrm{~nm}$, (e) $J_{\mathrm{ph}}$ versus $b$ of the RNWs with $a=100 \mathrm{~nm}$, (f) The photocurrent enhancement factor (PEF) versus $a$ of the RNWs compared to the SNW1 with the same $b=1000 \mathrm{~nm}$ and (g) PEF versus $b$ of the RNWs compared to the SNW2 with the same $a=100 \mathrm{~nm}$ for TM, TE, and unpolarized light, respectively; The representative normalized $G$ profiles of the RNWs with $a=1000,500,200,100 \mathrm{~nm}$ at a fixed $b=1000$ $\mathrm{nm}$ and $b=1000,500,200,100 \mathrm{~nm}$ at a fixed $a=100 \mathrm{~nm}$ for (h) TM and (i) TE light illumination (with an identical color scale), respectively. 


\section{Conclusions}

In summary, we presented the improved light absorption in the NWs based on symmetrybreaking structure from the square to the rectangular cross-section. We found that the light absorption in RNWs could be significantly improved in comparison with the SNWs and the enhancement effect mainly resulted from the symmetry-broken structure, which can simultaneously realize the increase of the light path length by the vertical side and the enhanced LMRs by the horizontal side. The simulation results showed that the photocurrent was significantly enhanced by $276.5 \%$ or $82.9 \%$ compared with that of the SNW with the same side length as the horizontal side of $100 \mathrm{~nm}$ or the vertical side of $1000 \mathrm{~nm}$, respectively. Therefore, the RNWs can be employed to other semiconductors to increase light absorption and provides an exciting pathway for the future development of high-efficiency single NW solar cells.

Funding: This research was funded by the National Natural Science Foundation of China (Grant No. 61404057, 51801068, and 11875284), Key disciplines Fund for Optical Engineering in Henan Province (Grant No. 2018119), and Key Youth Teacher of Universities in Henan Province (Grant No. 2016GGJS-145).

Acknowledgments: We would like to thank the China Scholarship Council (Grant No. 201902720047) and the Master's Program on Energy and Power Engineering at Huanghuai University.

Conflicts of Interest: The authors declare no conflict of interest.

\section{References}

1. Tian, B.; Zheng, X.; Kempa, T.J.; Fang, Y.; Yu, N.; Yu, G.; Huang, J.; Lieber, C.M. Coaxial silicon nanowires as solar cells and nanoelectronic power sources. Nature 2007, 449, 885-889, doi:10.1038/nature06181.

2. Nehra, M.; Dilbaghi, N.; Marrazza, G.; Kaushik, A.; Abolhassani, R.; Mishra, Y.K.; Kim, K.H.; Kumar, S. 1D semiconductor nanowires for energy conversion, harvesting and storage applications. Nano Energy 2020, 76, 104991, doi:10.1016/j.nanoen.2020.104991.

3. Li, Z.; Tan, H.H.; Jagadish, C.; Fu, L. III-V Semiconductor Single Nanowire Solar Cells: A Review. Adv. Mater. Technol. 2018, 3, 1800005, doi:10.1002/admt.201800005.

4. Kempa, T.J.; Day, R.W.; Kim, S.-K.; Park, H.-G.; Lieber, C.M. Semiconductor nanowires: a platform for exploring limits and concepts for nano-enabled solar cells. Energy Environ. Sci. 2013, 6, 719-733, doi:10.1039/C3EE24182C.

5. Christesen, J.D.; Zhang, X.; Pinion, C.W.; Celano, T.A.; Flynn, C.J.; Cahoon, J.F. Design principles for photovoltaic devices based on Si nanowires with axial or radial p-n junctions. Nano Lett. 2012, 12, 6024-6029, doi:10.1021/nl303610m.

6. Zhan, Y.; Li, X.; Li, Y. Numerical Simulation of Light-Trapping and Photoelectric Conversion in Single Nanowire Silicon Solar Cells. IEEE J. Sel. Top. Quantum Electron. 2013, 19, 1-8, doi:10.1109/jstqe.2013.2246771.

7. Tian, B.; Kempa, T.J.; Lieber, C.M. Single nanowire photovoltaics. Chem. Soc. Rev. 2009, 38, 16-24, doi:10.1039/B718703N.

8. Tang, J.; Huo, Z.; Brittman, S.; Gao, H.; Yang, P. Solution-processed core-shell nanowires for efficient photovoltaic cells. Nat. Nanotechnol. 2011, 6, 568-572, doi:10.1038/NNANO.2011.139.

9. Cao, L.; White, J.S.; Park, J.-S.; Schuller, J.A.; Clemens, B.M.; Brongersma, M.L. Engineering light absorption in semiconductor nanowire devices. Nat. Mater. 2009, 8, 643-647, doi:10.1038/nmat2477.

10. Cao, L.; Fan, P.; Vasudev, A.P.; White, J.S.; Yu, Z.; Cai, W.; Schuller, J.A.; Fan, S.; Brongersma, M.L. Semiconductor Nanowire Optical Antenna Solar Absorbers. Nano Lett. 2010, 10, 439-445, doi:10.1021/n19036627.

11. Brönstrup, G.; Jahr, N.; Leiterer, C.; Csáki, A.; Fritzsche, W.; Christiansen, S. Optical properties of individual silicon nanowires for photonic devices. ACS Nano 2010, 4, 7113-7122, doi:10.1021/nn101076t. 
12. Kim, S.; Cahoon, J.F. Geometric Nanophotonics: Light Management in Single Nanowires through Morphology. Acc. Chem. Res. 2019, 52, 3511-3520, doi:10.1021/acs.accounts.9b00515.

13. Choi, J.S.; Kim, K.-H.; No, Y.-S. Spatially localized wavelength-selective absorption in morphologymodulated semiconductor nanowires. Opt. Express 2017, 25, 22750-22759, doi:10.1364/OE.25.022750.

14. Zhang, C.; Yang, Z.; Wu, K.; Li, X. Design of asymmetric nanovoid resonator for silicon-based singlenanowire solar absorbers. Nano Energy 2016, 27, 611-618, doi:10.1016/j.nanoen.2016.08.017.

15. Luo, S.; Yu, W.B.; He, Y.; Ouyang, G. Size-dependent optical absorption modulation of Si/Ge and Ge/Si core/shell nanowires with different cross-sectional geometries. Nanotechnology 2015, 26, 085702, doi:10.1088/0957-4484/26/8/085702.

16. Kim, S.-K.; Song, K.-D.; Kempa, T.J.; Day, R.W.; Lieber, C.M.; Park, H.-G. Design of Nanowire Optical Cavities as Efficient Photon Absorbers. ACS Nano 2014, 8, 3707-3714, doi:10.1021/nn5003776.

17. Kim, S.-K.; Day, R.W.; Cahoon, J.F.; Kempa, T.J.; Song, K.-D.; Park, H.-G.; Lieber, C.M. Tuning Light Absorption in Core/Shell Silicon Nanowire Photovoltaic Devices through Morphological Design. Nano Lett. 2012, 12, 4971-4976, doi:10.1021/nl302578z.

18. Kempa, T.J.; Cahoon, J.F.; Kim, S.-K.; Day, R.W.; Bell, D.C.; Park, H.-G.; Lieber, C.M. Coaxial multishell nanowires with high-quality electronic interfaces and tunable optical cavities for ultrathin photovoltaics. $P$. Natl. Acad. Sci. USA 2012, 109, 1407-1412, doi:10.1073/pnas.1120415109.

19. Liu, W.; Oh, J.I.; Shen, W.Z. Light Trapping in Single Coaxial Nanowires for Photovoltaic Applications. IEEE Electron Device Lett. 2011, 32, 45-47, doi:10.1109/LED.2010.2086428.

20. Liu, W.; Oh, J.I.; Shen, W.Z. Light absorption mechanism in single c-Si (core)/a-Si (shell) coaxial nanowires. Nanotechnology 2011, 22, 125705, doi:10.1088/0957-4484/22/12/125705.

21. Liu, W.; Guo, X.; Xing, S.; Yao, H.; Wang, Y.; Bai, L.; Wang, Q.; Zhang, L.; Wu, D.; Zhang, Y., et al. OffResonant Absorption Enhancement in Single Nanowires via Graded Dual-Shell Design. Nanomaterials 2020, 10, 1740, doi:10.3390/nano10091740.

22. Yang, Z.; Cao, G.; Shang, A.; Lei, D.Y.; Zhang, C.; Gao, P.; Ye, J.; Li, X. Enhanced Photoelectrical Response of Hydrogenated Amorphous Silicon Single-Nanowire Solar Cells by Front-Opening Crescent Design. Nanoscale Res. Lett. 2016, 11, 233, doi:10.1186/s11671-016-1447-0.

23. Yang, Z.; Li, X.; Lei, D.Y.; Shang, A.; Wu, S. Omnidirectional absorption enhancement of symmetry-broken crescent-deformed single-nanowire photovoltaic cells. Nano Energy 2015, 13, 9-17, doi:10.1016/j.nanoen.2015.02.007.

24. Zhang, C.; Yang, Z.; Shang, A.; Wu, S.; Zhan, Y.; Li, X. Improved optical absorption of silicon singlenanowire solar cells by off-axial core/shell design. Nano Energy 2015, 17, 233-240, doi:10.1016/j.nanoen.2015.08.017.

25. Shi, L.; Zhou, Z.; Huang, Z. The influence of silver core position on enhanced photon absorption of single nanowire a-Si solar cells. Opt. Express 2013, 21, A1007-A1017, doi:10.1364/oe.21.0a1007.

26. Zhou, J.; Zhang, Z.; Wu, Y.; Xia, Z.; Qin, X. Significantly enhanced coupling to half-space irradiation using a partially capped nanowire for solar cells. Nano Energy 2018, 45, 61-67, doi:10.1016/j.nanoen.2017.12.027.

27. Leahu, G.; Petronijevic, E.; Belardini, A.; Centini, M.; Sibilia, C.; Hakkarainen, T.; Koivusalo, E.; Rizzo Piton, M.; Suomalainen, S.; Guina, M. Evidence of Optical Circular Dichroism in GaAs-Based Nanowires Partially Covered with Gold. Adv. Opt. Mater. 2017, 5, 1601063, doi:10.1002/adom.201601063.

28. Mann, S.A.; Garnett, E.C. Extreme Light Absorption in Thin Semiconductor Films Wrapped around Metal Nanowires. Nano Lett. 2013, 13, 3173-3178, doi:10.1021/n1401179h. 
29. Jia, Y.; Qiu, M.; Wu, H.; Cui, Y.; Fan, S.; Ruan, Z. Theory of Half-space Light Absorption Enhancement for Leaky Mode Resonant Nanowires. Nano Lett. 2015, 15, 5513-5518, doi:10.1021/acs.nanolett.5b02044.

30. Khaled, A.; Hameed, M.F.O.; Rahman, B.M.A.; Grattan, K.T.V.; Obayya, S.S.A.; Hussein, M. Characteristics of silicon nanowire solar cells with a crescent nanohole. Opt. Express 2020, 28, 31020-31033, doi:10.1364/OE.397051.

31. Liu, W.; Wang, Y.; Guo, X.; Song, J.; Wang, X.; Yi, Y. Light Trapping in Single Elliptical Silicon Nanowires. Nanomaterials 2020, 10, 12121, doi:10.3390/nano10112121.

32. Palik, E.D. Handbook of Optical Constants of Solids; Academic Press: London, UK, 1985.

33. Kane, Y. Numerical Solution of Initial Boundary Value Problems Involving Maxwell's Equations in Isotropic Media. IEEE Trans. Antennas Propag. 1966, 14, 302-307, doi:10.1109/TAP.1966.1138693.

34. Taflove, A.; Hagness, S.C. Computational Electrodynamics: The Finite-Difference Time-Domain Method, 3rd ed.; Artech House: Norwood, MA, USA, 2005.

35. Ee, H.S.; Song, K.D.; Kim, S.K.; Park, H.G. Finite-Difference Time-Domain Algorithm for Quantifying Light Absorption in Silicon Nanowires. Isr. J. Chem. 2012, 52, 1027-1036, doi:10.1002/ijch.201200061. 